\title{
How to increase safety in complex systems - an ongoing project
}

\author{
Håkan Alm*, Anita Gärling, Sara Saellström Bonnevier and Mats Danielsson \\ *Ph.D., Professor at the Department of Human Work Sciences, Division of Engineering psychology, Lulea \\ University of Technology, Sweden
}

\begin{abstract}
The aim of this paper is to describe an ongoing project with the aim of improving safety in an organization working with maintenance and development of the railway infrastructure in Sweden. The first sub goal was to investigate the General Failure Types in the organization. Seminars and interviews, based on the Tripod Delta method, with 62 employees resulted in a description of these latent errors in the organization. Recommendations for an improvement toward a safety culture was suggested, action plans were formulated and, in some cases, implemented. A follow up study is planned in a two year perspective
\end{abstract}

Key words: Safety, complexity, Tripod Delta, maintenance, railway infrastructure

\section{Introduction}

Safety or lack of safety is a common problem for many organisations today. Incidents and accidents may cause personal as well as financial losses and should be avoided as much as possible. In large and complex organisations where people are interacting with technology incidents and accidents do occur. According to Perrow [1] complex systems will always produce surprises, sometimes in the form of incidents or accidents. An important task is to find strategies to eliminate or reduce incidents and accidents in organisations and to create a safety culture. According to Reason [2] a safety culture is an informed culture where safety problems are identified and discussed. It is a reporting culture where incidents and accidents are reported without fear of being punished and where there is a clear definition of acceptable behaviour. It is a just culture and people are not blamed for incidents or accidents that happen without intention. It is a flexible culture where the experience from employers is respected and used, and it is a culture where you learn from experience. The strategy used in this study to come closer to the ideal safety culture was based on the *

Tripod Delta method. Tripod Delta can be regarded as a safety philosophy and an integrated view on processes that may interfere with safe behaviour or encourage unsafe acts. A basic assumption in TripodDelta is that it is easier to change certain parts of the working environment and the organisation compared to changing people. Tripod-Delta is based on research in Human Factors and Ergonomics and has used knowledge from these disciplines to define eleven factors (General Failure Types or GFT;s) that may interfere with safe behaviour or encourage unsafe behaviour. These GFT; s can be regarded as latent errors in an organization and if they can be reduced or even eliminated it may be possible to prevent accidents and incidents from happening. By doing this it can be possible to work with a proactive approach instead of react after an incident or accident already has occurred.

\footnotetext{
* Corresponding author. e-mail: hakan.alm@1tu.se Phone:+46 $703242936,+46920491270$,
} 


\section{Objectives}

The overall goal of this project is to increase safety in an organisation (InfraNord) working with maintenance and development of railway infrastructure in Sweden. The interaction between people, heavy equipment and fast trains produce a dangerous environment where incidents and accidents do occur. The first sub goal was to analyse and describe the General Failure Types (GFT;s) in the organisation and suggest strategies to reduce the most important GFT;s

\section{Practice innovation}

The starting point of the project was eight seminars with employees in the organisation, including the steering committee. The aim of the seminars was to identify risks in the organisation, discuss each individual's perception of risks and choice of method(s) to cope with risky situations. During the seminars the risk concept was defined and discussed and the participants produced their own lists of risks in their own work situation. Discussions were held concerning attitudes to risk, psychological aspects of risk, unsafe behaviour, and safety culture. During these seminars the participants produced lists describing risky work tasks, risky behaviour, and perceived attitudes to risks in the organization. They also discussed safety culture and their organizations status in relation to an ideal model of safety culture.

In the next phase of the project the aim was to identify error producing factors in the organisation, as specified by the Tripod-Delta method. In total 62 employees aged $26-61$ were interviewed in order to get a deeper understanding of the different GFT; $\mathrm{s}$ in the organization. The interviews were structured but with one open question at the end where the subjects were free to add anything they wished. Three skilled interviewers participated and each employee was interviewed individually. Notes were taken during the interview and a tape recorder was used in nearly all (one exception) interviews. The participants answer to the different questions was later noted on separate documents (word files) for each participant. Each interview took between 40 - 120 minutes to perform.

\section{Findings}

The interviews were focussed on the eleven general failure types presented below:

- Equipment, design, quality and availability

- Maintenance, quality, safety, and efficiency

- Procedures for different tasks

- Rules and their adaptation to reality

- Error enforcing conditions

- Housekeeping

- Incompatible goals

- Communication

- Organisation

- Training

- Defences

\section{Equipment}

According to the answers the equipment used was regarded as safe to use, but in some cases old, heavy and broken. The usability of the portable computer used by some personnel was rated as low. The availability of equipment was not optimal, 47 percent answered that they had access to the equipment needed to do their job. To solve the problem different strategies were used, such as to borrow equipment from co workers, buy or rent equipment, search for or improvise. The availability of equipment was also a problem during lunch brakes, late evenings and night shift. To address these problems it was suggested that when new equipment is needed workers with experience of the job (at the sharp end) should be engaged. Training to use the handheld computers was also suggested.

\section{Maintenance}

The planning of maintenance was pointed out as a problem. The time available for maintenance work was reported as often being too short and groups were often undermanned. Administrative tasks had increased in number and took more and more time from the time needed to solve the problem at hand. It was recommended to form fixed groups composed of people who are used to work together and know each others competence, to design better descriptions of how the work should be performed, to increase the number of workers in the groups and to improve the coordination of 
the jobs. A recommendation was that the employees (leaders) responsible for planning activities should get factual experience from the demands of the job tasks being performed at the field. Also that group composition should be designed by employees that really know the expertise of different field workers.

\section{Procedures for different tasks}

Procedures exist according to 66 percent of the interviewed and were reasonable adapted to the different tasks. However some rules were impossible to follow if the job should be finished in time. Informal rules were said to exist and were created as shortcuts to fix the job in time. The rules could be improved and be better adapted to the real working situation and the actual time demands. An alternative would be to hire more people and reduce the workload for the groups. Better description on tolerance levels for different tasks was mentioned also.

\section{Rules for the performance of different tasks}

Rules exist but were often not adapted to task demands or local conditions. There exists a large variation in work conditions between parts of the railway system with a high traffic volume compared to parts where the traffic volume is much lower, but rules were not adapted to those different contexts. Administrative tasks had increased and took time which may produce time stress. Rules are often written in a complicated style and are hard to interpret. In many cases it is impossible to follow all rules and have time enough to do the job, often a conflict between safety and productivity is created. It was suggested to write rules more adapted to people without an education in law and to simplify and adapt rules to real conditions. Changes in terminology should be avoided.

\section{Error enforcing conditions}

Time pressure often results in shortcuts of different kind and ignorance of safety rules. Lack of personnel and information can also increase the risk of accidents. Complicated procedures are needed to get a track free from trains for the time work is being completed and problems associated with this situation was pointed out. A train watch must be used when trains still are operating on the track where work is being done.

\section{Housekeeping}

Old equipment, bad planning tools, lack of personnel, absence of participation, bad service of equipment and incomplete knowledge in the steering group was pointed out as old problems still around. A recommendation was to educate the steering group to increase their understanding of the demands facing employees at the frontline. Improved communication between the steering group and personnel in the field was recommended.

\section{Incompatible goals}

Safety is pointed out as essential but productivity and the threat from other organisations doing the same job creates a conflict between safety and productivity. Employees are loyal despite problems with too few field workers and time pressure It was recommended to provide more time to different tasks and to increase the number of field workers. The planning of different tasks should be improved.

\section{Communication}

Written information was sometimes unclear and difficult to interpret. A recommendation was to increase the trust between leaders and field workers and to reward employers when they point out safety problems. Simplified and clear face-to-face communication was recommended.

\section{Organization}

85 percent of the employers answered that clear rules exist for different roles in the organization. It was suggested that safety must be stressed by the top of the organization and that more time is needed to improve safety.

\section{Training}

67 percent of leaders and 47 percent of field workers answered that time and resources were 
available for training. Improved control of safety rules was suggested as well as better communication and more time and training using different scenarios was suggested.

\section{Defences}

All employees answered that defences or different barriers existed, but that lack of availability and bad or clumsy design of personal protection devices were problems. A bad attitude was also mentioned, for instance some employees were not using personal protection (helmets, eye protection) for different reasons.

For each GFT a separate list with proposals for improvement were produced, based on the interviews. The results from the interviews have been presented for the steering group and for the group of field workers. During these meetings comments and clarifications could be made.

\section{Discussion}

A common theme that emerged during the interviews was lack of personnel and time and also problems with the equipment used. Communication problems were also highlighted. Nearly all employees (100 percent of the leaders and 96 percent of fieldworkers) mentioned that incidents may occur without being reported. Fear of punishment, difficulties in the handling of the reporting system, unclear definition of the concept incident, were mentioned as possible reasons for this. A more clear definition of the concept incident, removal of fear of reporting, and a better system for incident reporting are possibilities to change this fact and move closer to a reporting culture in the organization. One main reason for the safety problems in the organization seems to be the pressure to create a good economic situation in the organization. The balance between productivity and safety and the competition from other organizations capable of doing the same job probably leads to a situation where productivity comes first and safety a bit behind. The pressure from productivity seems to create time pressure and an unwillingness to employ more personnel or costly investments in new and more advanced equipment.
Based on the information concerning the eleven GFT; s a number of action plans have been developed and some have been implemented. Work descriptions are being improved and safety problems are more often stressed by the leaders of the organization and will be discussed at every meeting as a separate question. The problem with a train reporting role and possible solutions have been discussed and a technical solution have been suggested and will be studied further. Group leaders have developed plans to participate in field work and learn more of the demands in different tasks. Some steps in the direction of a safety culture have been taken so far. Safety problems in the organization have been discussed and highlighted as important. The importance of reporting safety problems of different kind, incidents as well as accidents, have been stressed. Different strategies to increase reporting have been discussed and the importance of a just, flexible and learning culture has been stressed. One field worker has been recruited to work with safety questions.

A follow up study is planned to start in about two year time and will provide a possibility to evaluate the effects of this project.

\section{References}

[1] Perrow, C. (1999). Normal Accidents - Living with High-Risk Technologies. Princton University Press.

[2] Reason, J. (1997). Managing the risks of Organizational Accidents. Ashgate 